Ann. Génét. Sél. anim., I971, 3 (4), 425-432.

\title{
DÉTERMINISME HÉRÉDITAIRE DE LA COULEUR BLANCHE DE LA GHEVRE SAANEN
}

\author{
G. RICORDEAU et J.-J. LAUVERGNE \\ avec la collaboration technique de P. Guillimin \\ Laboratoire de Génétique des Petits Ruminants, \\ Centre de Recherches de Toulouse, I. N. R. A., \\ B. P. 12, 31 - Castanet \\ Laboratoire de Génétique factorielle, \\ Centre national de Recherches zootechniques, I. N. R. A., \\ 78 - Jouy-en-Josas
}

\section{RÉSUMÉ}

En 1969 et r970 au Domaine expérimental de Galle par Avord (Cher) on a fait des croisements de première génération et de retour entre des chèvres Saanen blanches et des chèvres alpines chamoisées ou poitevines.

En $F_{1}$ les animaux des deux sexes sont presque tous blancs (comme le Saanen) ou rouans (402 sur 406). Le back-cross sur le parent coloré donne 88 animaux colorés pour 79 animaux de type blanc ou dilué des deux sexes.

Ainsi le déterminisme du blanc apparaît comme monofactoriel autosomal, dominant. Sı les cas de $\mathrm{F}_{1}$ de type alpin observés ne sont pas des erreurs, la pénétrance serait subtotale : $99 \mathrm{p}$. Ioo.

En $\mathrm{F}_{1}$ les mâles sont, en moyenne, plus foncés que les femelles à la naissance, mais la différence n'est pas significative. En revanche, la nuance rouanne s'assombrit significativement avec l'âge entre la naissance et un an, chez les femelles tout au moins. On n'a pas de données pour les mâles.

L'action de ce gène dominant est totalement épistatique sur toute autre coloration à l'état homozygote et chez une partie des hétérozygotes. Chez les rouans on note une dilution qui semble plus marquée sur la mélanine noire (eumélanine) que sur la mélanine rouge (phœomélanine). L'assombrissement observé chez les back-cross de type blanc ou dilué par rapport aux $F_{1}$ montre qu'une sélection polygénique a pu intervenir pour faire varier l'expressivité du facteur. On propose pour ce gène le nom de Rouan (symbole $R$ pour l'allèle rouan, $r$ pour l'allèle normal).

La race de chèvres Saanen ( $\left.{ }^{1}\right)$ est originaire de Suisse où elle occupe en gros la moitié ouest du pays. En I966, avec une vingtaine de milliers de têtes (BUREAU FÉDÉRAL, DE STATISTIQUE, I968) elle constituait environ le quart du cheptel caprin helvétique (ANONYME, a).

(1) En Suisse romande on dit aussi chèvre de Gessenay, appellation qui ne semble guère avoir franchi les limites de la Confédération. 
Le standard coloré de cette race à poil court est le blanc uniforme. Comme le fait déjà remarquer CRÉPIN (Igo6) il s'agit là d'une couleur existant certainement depuis longtemps dans les populations caprines des Alpes franco-suisses. D'ailleurs il y a, en Suisse, une autre race, au sens zootechnique moderne du terme, qui a le même standard coloré : la race d'Appenzell (ANONy ME, b).

Les études jusqu'alors consacrées au déterminisme héréditaire de la couleur blanche de la Chèvre sont peu nombreuses ( 4 au total) et anciennes pour la plupart (I926, I928, I94I, I966). A partir de résultats provenant du Domaine expérimental de Galle par Avord (Cher), Institut national de la Recherche agronomique, nous voudrions vérifier et préciser leurs conclusions ( ${ }^{1}$ ).

\section{MATÉRIEI ETT MÉTHODES}

Du côté femelle on est parti de deux souches : une souche colorée comprenant essentiellement des chèvres de race alpine chamoisée et quelques chèvres poitevines ou croisées chamoisée $\times$ Poitevine; une souche blanche comprenant 80 chèvres de race Saanen originaires de Suisse et inscrites au Herd-book de la race, plus 3 chèvres blanches appartenant à la même variété élevée en France.

Tous les boucs colorés étaient alpins chamoisés à ventre noir. Les mâles blancs étaient tous des Saanen provenant, comme les 80 femelles déjà citées, d'un lot importé de Suisse.

Les accouplements entre mâles blancs et femelles colorées ainsi que les accouplements réciproques ont eu lieu en 1969 et en 1970 . Les croisés de première génération $\left(F_{1}\right)$ sont nés en 1970 et I97I. Les croisés de retour sont tous nés en I97 I. On a réalisé les deux back-cross : Saanen $\times F_{1}$ et surtout alpin chamoisé $\times \mathrm{F}_{1}$ et croisement réciproque.

Une partie des descendants observés, $F_{1}$ ou back-cross, ont été photographiés, ce qui a permis d'étudier avec plus de précision la variation de la dilution que l'on observe dès la $F_{1}$. On a utilisé pour cela une échelle simple à 4 degrés :

o : blanc (rappelant le parent blanc Saanen),

I : traces de rouan,

2 : rouan plus ou moins intense et étendu,

3 : coloré (rappelant le parent coloré chamoisé ou poitevin).

Les croisés de retour ont tous été examinés visuellement et, pour la plupart des femelles, photographiés.

\section{RÉSULTATS}

\section{A. - Résultats globaux des croisements}

Les résultats des observations des $F_{1}$ et des deux back-cross sont donnés dans le tableau I.

\section{B. - Variation de la dilution}

La notation à partir des diapositives en couleur permet de comparer un certain nombre de populations différant par le sexe, l'âge ou la génération. Les résultats globaux sont donnés dans le tableau 2.

(1) L'essentiel des résultats de cet article a été présenté à la $I{ }^{\mathrm{e}}$ Conférence internationale des Éleveurs de Chèvres, Tours, le I 8 juillet I97I. 
COULEUR BLANCHE DE LA CHÈVRE " SAANEN "

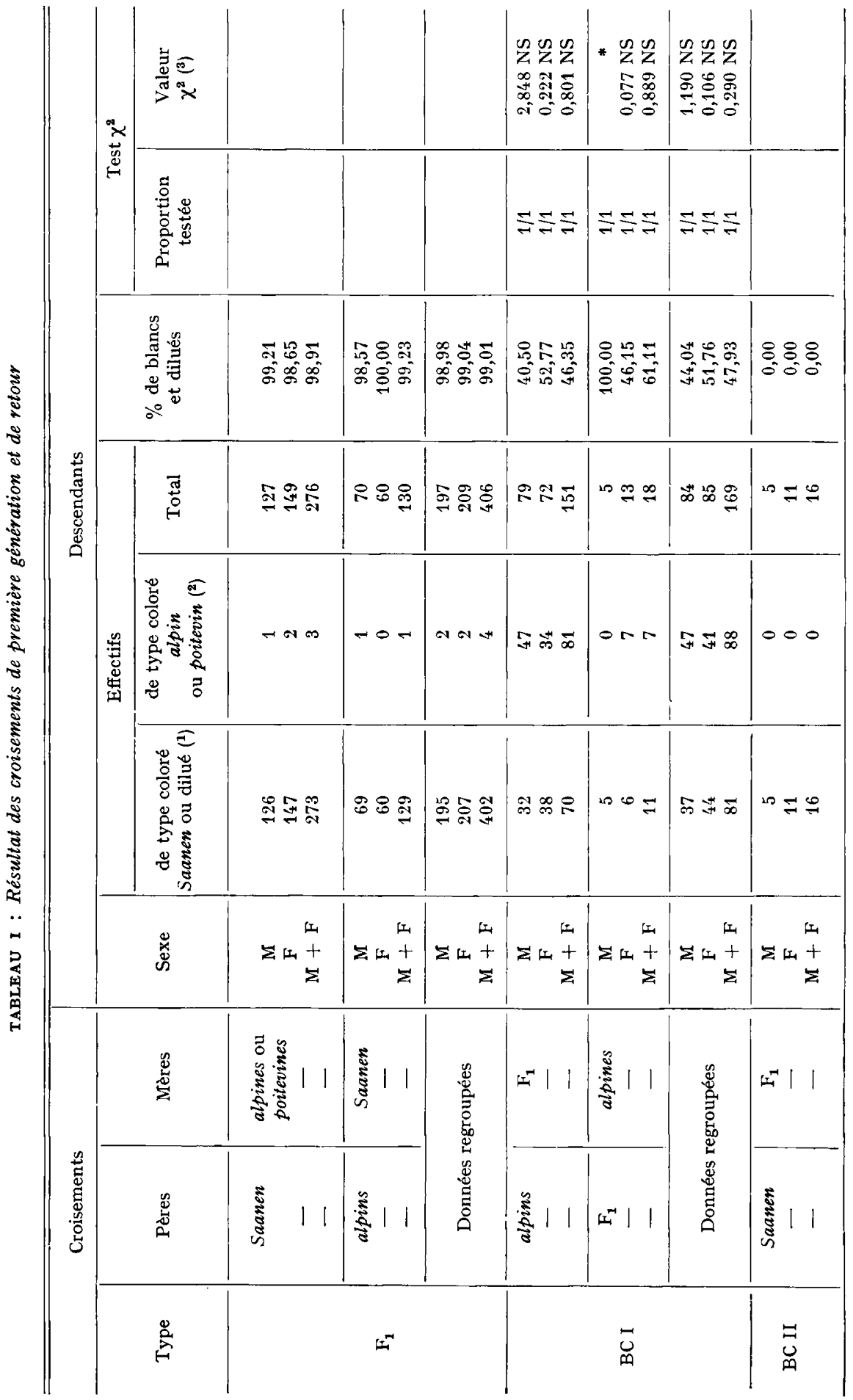

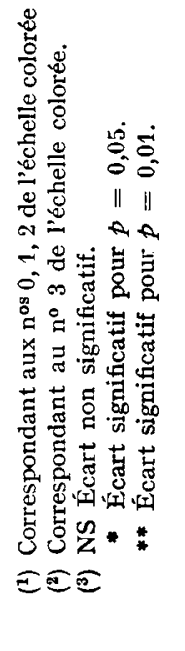


G. RICORDEAU, J.-J. LAUVERGNE

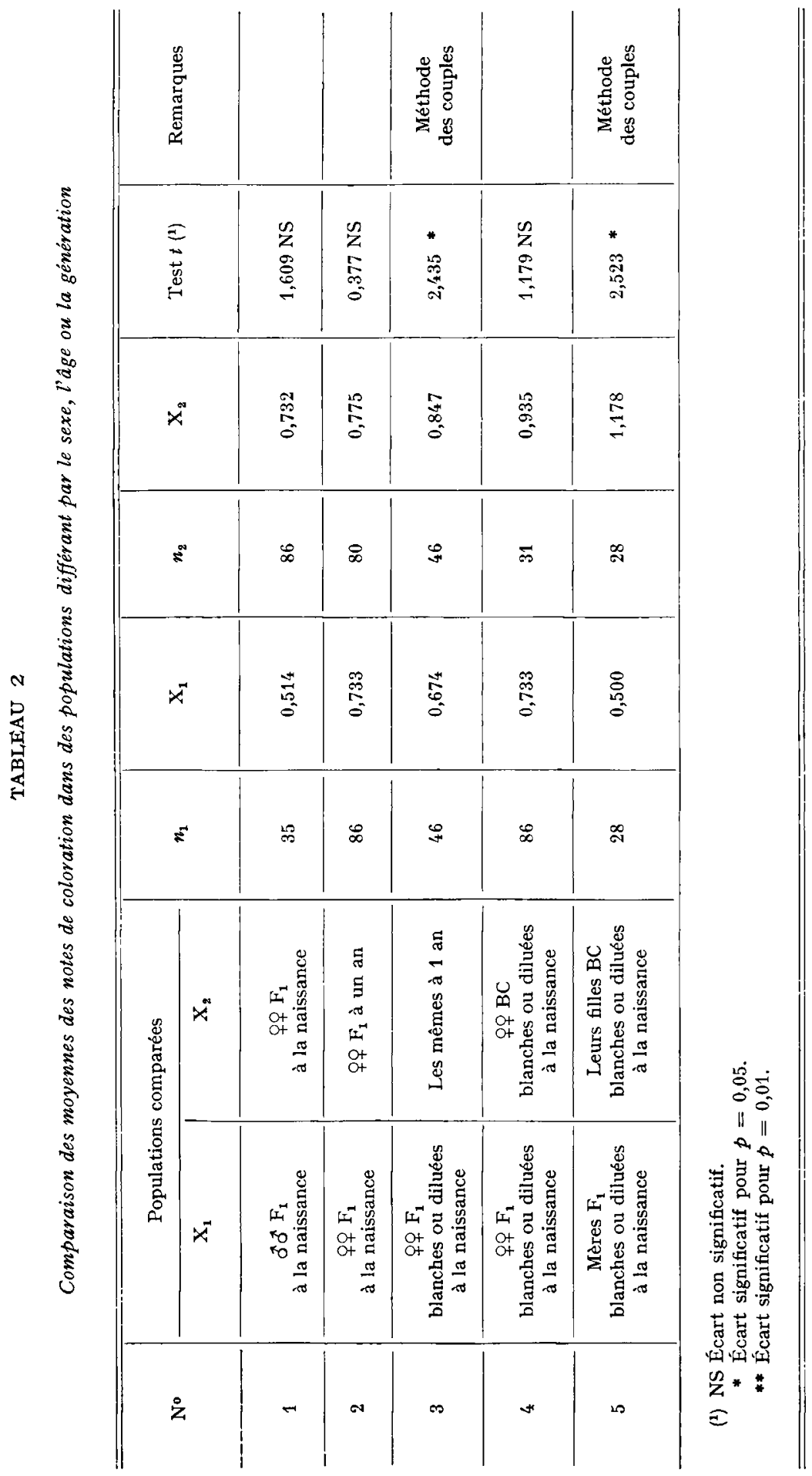


Il est à noter que, si les plages rouannes qui apparaissent chez les $F_{1}$ et les backcross ne couvrent en général qu'une faible partie du corps de l'animal, elles le font symétriquement. On peut de ce fait distinguer l'action de ce gène de celle du facteur de panachure irrégulière également présent dans notre population.

L'action de dilution semble assez poussée sur la mélanine noire présente si typiquement sur la raie dorsale et sur le ventre des chèvres chamoisées. La phœomélanine rouge reste le plus souvent seule en coloration atténuée.

Aussi bien par la superficie des taches, par leur nuance que par le nombre d'animaux très dilués, les $F_{1}$ et les back-cross de type $F_{1}$ se rapprochent beaucoup plus du parent blanc Saanen que du parent coloré alpin ou poitevin comme l'indiquent les moyennes du tableau 2 et comme l'illustrent les histogrammes de la figure $I$.

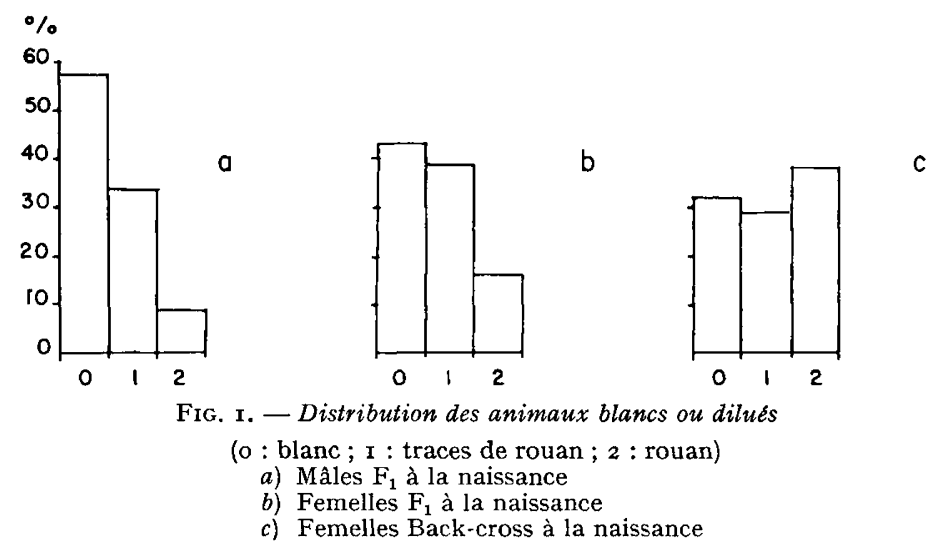

\section{DISCUSSION}

\section{A. - Le déterminisme héréditaire proprement dit}

Du tableau I il ressort clairement que la couleur blanche de la race Saanen est commandée par un gène autosomal dominant l'allèle qui autorise la coloration normale de l'espèce.

Si, chez l'homozygote, l'action du gène qui donne le blanc semble totale, par contre, chez l'hétérozygote, on voit apparaître, à côté d'animaux qui ressemblent au parent dominant, des individus plus ou moins dilués. Il y a donc dominance incomplète et, si l'expressivité du gène varie, certains hétérozygotes à la limite pourront se confondre avec le parent intensément coloré. La pénétrance chez l'hétérozygote ne sera alors plus totale. C'est de cette manière que l'on peut expliquer les quelques exceptions du tableau I qui, après vérifications poussées, ne semblent pas relever d'erreurs lors de la monte ou de 1'identification des produits. Quoi qu'il en soit, la pénétrance reste élevée ( $99 \mathrm{p}$. I00) ce qui est conforme avec le pourcentage important d'hétérozygotes indiscernables de 1'homozygote tout blanc (fig. I).

L,USH (I926) travaillant sur la race Toggenburg aux États-Unis étudie séparément le blanc, le rouan et le "bleu ". Il conclut qu'il est difficile de préciser le déter- 
minisme héréditaire du blanc. ASDELL et BUCHANAN-SMith (I928) concluent, quant à eux, que le blanc est de type dominant et qu'au moins la couleur crème pourrait être une forme d'expressivité de ce facteur. Ils continuent néanmoins à traiter indépendamment le gris et le rouan. EIDRIGEvic (I94I), qui analyse des données soviétiques, pense aussi qu'il y a un type monofactoriel de blanc à comportement épistatique sur les autres couleurs, mais il ne s'emploie pas, lui non plus, à analyser les modes d'expressivité de ce facteur. BERGE (I966) enfin, tout en ajoutant quelques données norvégiennes à celles qu'il compile chez L,ush (I926), se montre assez peu clair sur 1'interprétation génétique de la couleur blanche.

Ainsi SEARLE (I968) dans son ouvrage de synthèse sur la coloration des Mammifères peut-il s'étonner de la présence d'une couleur crème qui serait génétiquement distincte du blanc. Cet auteur pense que le rouan doit représenter, comme dans d'autres espèces, une condition hétérozygote. Nos résultats semblent confirmer cette manière de voir.

Si les conclusions des anciens auteurs manquent de consistance pour expliquer le déterminisme du blanc, par contre, sur un point au moins leurs observations doivent retenir notre attention : l'existence d'animaux "bleus " qui peuvent résulter de l'action dudit gène sur des phénotypes noirs que malheureusement nous n'avions pas dans nos souches de départ. Dans certains cas, il se peut que la disparition de la mélanine noire ne soit pas aussi poussée que nous avons pu le constater.

\section{B. - Interprétation de la variation dans l'expressivité: la possible action de la sélection}

Une première source de variation de la dilution pourrait être le sexe. Nos données en nombre assez réduit il faut le dire ne nous permettent pas de mettre en évidence une telle action, au moins à la naissance (tabl. 2, II).

Vient ensuite 1'âge dont, au moins chez les femelles, l'action est significative quand on fait les comparaisons par couples. Les animaux deviennent plus foncés en vieillissant (tabl. $2: 2,3$ ).

Quant à l'action de facteurs génétiques on peut la rechercher en comparant la couleur moyenne des descendants de type rouan en $F_{1}$ puis en back-cross. On note, alors, au moins dans les comparaisons par couples mère-fille (tabl. 2, 5), que les back-cross sont significativement plus foncés que les $F_{1}$. Cela peut s'expliquer par une diminution, à chaque génération de croisement avec un parent coloré, du stock de polygènes de la souche blanche. On se trouverait ainsi dans une situation analogue à celle du mouton berrichon blanc analysée par l'un d'entre nous (LAUVERGNE, I969). Dans cette race ovine, on montrait que la parfaite couleur blanche avait été obtenue à partir d'un gène de panachure à dominance incomplète et expressivité variable dont les éleveurs avaient, par sélection, modifié le comportement.

\section{C. - Terminologie}

Pour le moment le terme de rouan n'a qu'une valeur descriptive superficielle; il ne préjuge en rien de l'action intime du facteur, sur la migration du mélanocyte, sa forme, sa densité et son activité prise dans son sens le plus large (production d'une mélanine modifiée en quantité ou en qualité). On peut cependant au moins provi 
soirement le proposer pour désigner ce gène avec les symboles $R$ pour l'allèle dominant $r$ pour l'allèle normal.

L'homologie phénotypique la plus facile avec d'autres espèces est sans doute celle avec le blanc des Bovins charolais. Dans cette race dont le standard coloré est le blanc pur on observe en effet des animaux dilués (rouans ou gris) en première génération de croisement avec une race normalement colorée (LAUVERGNE, I966).

\section{CONCLUSION}

L'analyse génétique de la couleur blanche de la Chèvre se rattache à l'étude plus générale des gènes à pénétrance incomplète et à expressivité variable qui ont été soumis par l'Homme à une sélection intense en raison de l'intérêt esthétique que la coloration en question présentait à ses yeux.

Un tel mode de sélection présente évidemment un certain danger car la pression énorme de sélection en faveur des génotypes qui plaisent peut aisément masquer des défauts coûteux pour l'élevage. Les exemples ne manquent pas, précisément avec le blanc, dans l'espèce bovine tout au moins; blanc du Shorthorn (RENDEL, I952) et blanc de la race des Montagnes de Suède (LAuvergne, I970). Bien qu'il faille se garder d'une généralisation hâtive il est tout de même curieux de noter qu'EidnIGEVIC (I94I) relevait chez les chèvres blanches un taux de mortalité juvénile supérieur à celui des chèvres diversement colorées. Vue l'extension actuelle de la race Saanen une vérification de cette assertion semble s'imposer, de même qu'il faudrait contrôler une éventuelle action sur d'autres paramètres de production et de reproduction.

Reçu pour publication en novembre 1971.

\section{REMERCIEMENTS}

Le Dr A. G. Searle (Medical Radiobiological Unit, Harwell Didcot, Berks., Angleterre) a bien voulu relire ce manuscrit suggérant d'utiles corrections.

\section{SUMMARY}

\section{HEREDITARY WHITE COLOR DETERMINISM IN THE SAANEN GOAT}

In 1969 and 1970 at the Galle Experimental Domain, I 8 - Avord par Bourges (Cher), firstgeneration and back-crosses between white Saanen and chamois-alpine or Poitou goats were made.

The $F_{1}$ animals of both sexes were almost all white (as the Saanen) or roan (402 out of 406 The back-cross on the colored parent gave 88 animals to 79 white or off-white animals of both sexes.

White determinism thus appears as a monofactorial, autosomal dominant. If the cases observed of alpine $\mathrm{F}_{1}$ type are not mistakes, penetrance would be subtotal or $99 \mathrm{p}$. Ioo.

On the average, $F_{1}$ males are darker than the females at birth but the difference is not significant. On the other hand, the roan shade darkens whith age between birth and one year, at least in the females. There is no data on males. 
The action of this dominant gene is completely epistatic on all other coloring in the homozygote state and in some heterozygotes. In roans, there is an off-shade which seems more marked for black melanin (eumelanin) than for red melanin (phoeomelanin). The darkening observed. in the white or off-white back-crosses as compared to the $F_{1}$ showed that polygenic selection could make the expression of the factor vary. We propose to call this gene Roan: $\mathrm{R}$ being the roanning allele and $r$ the normal one.

\section{RÉFÉRENCES BIBLIOGRAPHIQUES}

Anonyme, a. L'élevage caprin et la garde des chèvres en Suisse. La terre et la forêt, présentation du bétail, élevage du menu bétail, Caprins, 66,3/1. Brochure fédérale.

Anonyme, $b$. Description des races de menu bétail. Recensement fédéral du bétail, 21 avril 1966. Formule 4 p., I I 876.

Asdell S. A., Buchanan Smith A. D., r928. Inheritance of color, beard, tassels and horns in the goat. J. Hered., 19, 425-430.

Berge S., 1966. Geitefarger. Meld. Norges Landbrukhgösk, 45 (3), I-24.

Bureau fédéral de Statistique., 1968. Le Cheptel suisse rg66. Statistiques de la Suisse, $42 \mathrm{I}^{\mathrm{e}}$ fasc. série ch 8, Berne.

CREPIN J., Igo6. La Chèvre, son histoire, son élevage pratique, ses bienfaits, ses services. Hachette, Paris.

EIDRIgevic E. V., I94I. A contribution to the problem of morphogenesis and evolution of pigmentation in the goat. Trud. Burjat. Mongolsk zoovet. Inst. (Ulan-Ude), 2, r73-187. (Abstr. in Anim. Breed. Abstr., 1946, 14, 237).

Lauvergne J. J., rg66. Génétique de la couleur du pelage des Bovins domestiques (Bos taurus, LinnÉ). Bibliogr. Genet., 20, r-68.

LAUVERGNe J. J., I969. Hérédité de la couleur blanche du mouton berrichon croisé à des solognots. Ann. Génét. Sél. anim., 1, 219-226.

LaUvergne J. J., 1970. Gonadal hypoplasia and white color in Swedish Highland cattle. J. Hered., 61, 43-44.

Lush J. L., 1926. Inheritance of horns, wattles and colors in grade Toggenburg goats. J. Hered., 17, 73-9I.

RENDEL J., 1959. White heifer disease in a herd of dairy Shorthorn. J. Genet., 51, 89-94.

Searle A. G., r968. Comparative Genetics of Coat colour in Mammals. Logos Press, Academic Press, London, New York, I95. 